\title{
Omental Endometriosis: A Rare Site and an Unusual Association with Ovarian Fibroma and Haemorrhagic Ascites. A Case Report And Review of The Imaging Techniques
}

\author{
A .O. Akintomide ${ }^{1}$, D. E. Bassey ${ }^{1}$, E. I. Ekanem ${ }^{2}$, A . J. Omotosho ${ }^{3}$. \\ ${ }^{1}$ Department of Radiology, University of Calabar, Calabar, Cross-River State, Nigeria). \\ ${ }^{2}$ Department of Obstetrics and Gynaecology, University of Calabar, Calabar, Cross-River State, Nigeria). \\ ${ }^{31}$ Department of Pathology, University of Calabar, Calabar, Cross-River State, Nigeria).
}

\begin{abstract}
A case of omental endometriosis with recurrent haemorrhagic ascites in a 22 year old nullipara is presented. She first presented at a private hospital with a three month history of progressive abdominal distention and the transabdominal ultrasound scan revealed a right ovarian mass with massive ascites. She had laparotomy and the histology reports of the omental and ovarian samples revealed endometriosis and fibroma respectively. She presented to us with a recurrence of the distension six months later. Repeat transabdominal ultrasound scan and an abdominopelvic computed tomograhpy revealed only a massive ascites. She had a second laparotomy and the histology of the degenerating omental sample still revealed endometriosis. Patient was managed satisfactorily on hormonal therapy without recurrence over a two year follow up. The Literature on the appropriate imaging modalities in endometriosis is reviewed and these showed that with judicious use, imaging is accurate in the pre operative diagnosis.
\end{abstract}

Keywords: Haemorrhagic ascites, Imaging, Omental endometriosis, Ovarian fibroma.

\section{Introduction}

Endometriosis is the presence of a tissue histologically similar to the endometrial tissue at sites outside the normal uterine cavity. It is a condition found at multiple sites among which the omentum is rare. The diagnosis of endometriosis requires a thorough investagation to localise and there's still a delay in arriving at this even in developed countries. The invasive diagnostic laparoscopy is the "gold standard" of investigation but radiological imaging is also accurate and is actually superior at a few specific sites. Ultrasonography (US) and Magnetic resonance imaging (MRI) have been found to be accurate in the pre-operartive diagnosis of endometriosis when their findings were correlated with those of laparoscopy and surgery. The two imaging modalities are non-invasive and non-ionsing. Computed tomography (CT) is ionising and occasional helpful but often yield non-specific findings. Imaging was not effectively utilized in this case especially at the initial presentation, largely because as of then, there were no functional CT and MRI machines in Calabar. Our laparoscope was also bad at that time.

We report this rare case of omental endometriosis co-existing with ovarian fibroma, which also had an unusual presentation of haemorrhagic ascites. This is to remind us of the possibility of multiple pathologies in an individual and to raise the awareness that endometriosis is an important differential diagnosis in haemorrhagic ascites in females in the child bearing age. The importance of using the appropriate imaging modality and technique to make an accurate diagnosis is also emphasized.

\section{Case Report}

Miss A. B a 22 year old Nigerian nullipara first presented at a private hospital with a three months history of progressive abdominal distension. The transabdominal Ultrasound scan(TAS) revealed massive ascites with internal echoes and a right ovarian mass which measured $3.25 \times 3.58 \mathrm{~cm}$ (Fig. 1).The mass was hypoechoic with a thick smooth wall and had 2 small nodules within (Fig. 2). An impression of ovarian fibroma with haemorrhagic ascites was made. Baseline laboratory investigations showed moderate red blood cells and bilirubin in the urine. The serum electrolyte, urea and creatinine, liver function test and full blood count were within normal limits. The bloody ascitic fluid obtained by abdominal paracentesis had a total protein of $10.8 \mathrm{mg} / \mathrm{dl}$ but did not contain malignant cells or acid fast bacilli. Retroviral screening was negative.

Subsequently, the patient underwent open laparotomy. The findings were 2.1 litres of haemorrhagic peritoneal fluid, chocolate coloured beaded omentum, a right ovarian mass and the descending colon was adherent to the uterus. Patient had partial omentectomy, the ascitic fluid was drained and the right ovarian mass debulked. The specimen of the omental nodules and right ovarian mass sent for histology revealed omental endometriosis and ovarian fibroma respectively. She was discharge home and placed on some oral drugs but can't recall the names. 
Six months post operation, she presented to us with recurrence of the abdominal swelling. A repeat transabdominal US showed a massive ascites with internal echoes and a right adnexal fullness. An abdominopelvic CT done revealed massive ascites with normal intraabdominal organs and lymphatic channels(Fig. 3).No mass was seen in the pelvis but the fundus of the uterus was displaced to the left relative to the bladder (Fig. 4). Due to the inconclusive findings, we carried out another open laparotomy and this showed degenerating omentum, severe adhesions of the pelvic organs and 5.9litres of haemorrhagic ascites. The sample of omentum was again sent for histology and this still revealed endometriosis. She was then given hormonal therapy (Danazol) with satisfactory response and no recurrence over a two year follow-up period.

\section{Literature review}

Massive haemorrhagic ascites is an unusual presentation of endometriosis and it is known to recur in some cases depending on the mode of treatment [1]. This presentation was first described by Brews in 1954 [2] with only 22 cases reported in the English literature as at 1995 [3]. It occurs predominantly in blacks [4]. In the reviewed literature, there was no report of endometriosis co-existing with ovarian fibroma but there were cases of synchronous primary ovarian neoplasm which led to the speculation that endometriosis might be a premalignant lesion [5,6]. In many developed countries, specialist gynaecologist see numerous patients in whom there has been an average delay time between the onset of symptoms and diagnosis of six to seven years due to wrong diagnosis by the general practitioner [7]. The "gold standard" for pre-opertive diagnosis is laparoscopy and often with a peritoneal biopsy. For the inexperienced surgeon, this invasive procedures are sometimes limited based on the atypical appearances of peritoneal lesions, particularly in adolescents or due to the deep and partially hiddenlesions (bladder, bowel and posterior pelvis). Radiological investigations of endometriosis are not invasive and they give accurate diagnosis when the right choice of modality and technique are made.

\subsection{Ultrasonography}

This is a non-ionising modality which is readily available. The pelvic assessment can be done using the transabdominal, transvaginal or transrectal approach. Transvagina sonography (TVS) is able to detect ovarian endometrioma provided it is greater tha $2 \mathrm{~cm}$ [8]. In the study of 81 patients in which a comparison was made between the accuracy of TVS and rectal endoscopic sonography (RES) in the diagnosing deep infiltrating endometriosis, TVS was more accurate than RES in specific location [9]. Compared to MRI, endoscopic ultrasound has better sensitivity close to $100 \%$ for the diagnosis of rectosigmoid endometriosis [10]. Endoscopic ultrasonography is a simple and non-invasive technique capable of correctly diagnosing rectal wall infiltration in deep pelvic endometriosis. It may be helpful in determining the choice between laparoscopy and laparotomy when complete resection is indicated [11]. Ultrasound is however limited in sensitivity for posterior lesions [12]. 3Dimensional (3D) US and power Doppler allow characterisation of the surface features, precise depiction of the size and volume, as well as the vasculature of cystic ovarian tumour [13].

The specific ultrasound features of endometrioma are a cyst with diffuse low level internal echoes and hyperechoic wall foci $[11,12]$. The later is said to be the single predictor of endometrioma and is thought to represent cholesterol deposit [14]. These wall foci are more echogenic and smaller than nodules. Other features are multilocularity which is commoner and more diagnostic than unilocularity, obliteration of the cul de sac, septations especially when thick, wall nodularity and rarely anaechoic cyst [12]. Adhesion is also a feature which presents as fixed retrovertion of the uterus even on external pressure. On colour flow Doppler ultrasound, endometrioma usually show scanty pericytic flow, especially noticeable in the hilar region and usually visualised in regularly spaced vessels [15].

\subsection{Abdominopelvic MRI}

MRI is a non-ionising radiological modality of investigation with superior soft tissue differentiation and multiplanar capability. This provide diagnosis of endometriosis in anterior and posterior lesions and, it enables complete lesion mapping prior to surgery [15]. It is used when US is indeterminate and can resolve lesions as small as $3 \mathrm{~mm}$. It does not improve the sensitivity and specificity of diagnosing ovarian endometrioma over US. It however has a higher sensitivity and likelyhood ratios for uterosacral ligament and vaginal endometriosis compared with TVS and RES [16]. A number of techniques have been developed to increase the sensitivity of MRI to detect pelvic endometriosis. These include the use of endovagina and rectal contrast which helps to better delineate the anatomy of interest and map out the disease. Hottat $\mathrm{N}$ et al filled the rectum with ultrasound gel before scanning with 3.0T MRI machine [17] . This was found to have a sensitivity and specificity of $96.3 \%$ and $100 \%$ respectively for colonic infiltration. Karen Kinkel et al gave I.V contrast and peristalsis inhibitors for easier identification of the hyperintense spots seen on $\mathrm{T}_{1} \mathrm{~W}$ image in posterior lesions [12]. Fat suppressed $\mathrm{T} 1$ weighted $\left(\mathrm{T}_{1} \mathrm{~W}\right)$ sequence was also found to be better than the conventional MRI [18].

The lesion is typically hyperintense on $T_{1} \mathrm{~W}$ with reduction in the intensity on $\mathrm{T} 2$ weighted $\left(\mathrm{T}_{2} \mathrm{~W}\right)$ images(shading). Another typical feature is the presence of multiple hyperintense cysts on $\mathrm{T}_{1} \mathrm{~W}$ regardless of the $\mathrm{T}_{2} \mathrm{~W}$ signal [19]. Peritoneal implants initially are small serosa lesions and usually escape detection. Larger, 
fibrotic peritoneal implants of endometriosis are seen $\mathrm{T}_{2} \mathrm{~W}$ images as spiculated nodules of very low signal intensity. Adhesions presents with spiculated low signal intensity stranding that obscure organ interface.

\subsection{Abdominopelvic CT}

It is an ionising imaging modality that generate cross sectional axial images with reconstruction in coronal and sagittal planes. This may constitute another potential option as a complementary imaging modality for the evaluation of deep pelvic endometriosis. CT-based virtual colonoscopy is a very useful tool in colorectal endometriosis. Vassilieff et al insufflated air into the distal rectum and gave intravenous contrast agent before 3D reconstruction of images to improve the visualisation of the lumen [20]. Multidetector Computed tomography with multiplanar reformation allows accurate examination of the complex anatomy of the peritoneal cavity and is a primary diagnostic method for evaluating the omenta [21].

The most specific findings of deep infiltrating endometriosis are tethered rectum, stranding of the periuterine pelvic fat, thickening of the uterosacral ligament and retroflexed uterus [20].

\section{Discussion}

The patient had only transabdominal US with a $3.5 \mathrm{MHz}$ curvilinear transducer at the first presentation. This was unable to detect the omental endometriotic nodules and the adhesions were not clearly resolved. The right ovarian cyst and massive ascites were however well outlined. The abdominal CT carried done at the second presentation was valuable, detecting the ascites and a right adnexal fullness with normal abdominopelvic organs, blood vessels and lymphatic channels. Abdominal CT is of value in omental anatomy and it would have detected the omental nodules if it was done prior to the first surgery. Though the US feature of pelvic adhesion is a fixed retroverted uterus, this was not observed in our patient but she rather had a right adnexal fullness which correlated with the CT findings. Though TVS and RES are superior to TAS and abdominal CT for deep endometriosis, they wouldn't have been able to detect the omental nodules or clearly delineate the adhesion. In the index patient, abdominal MRI would have been the appropriate modality to detect all the lesions and accompaning adhesions.

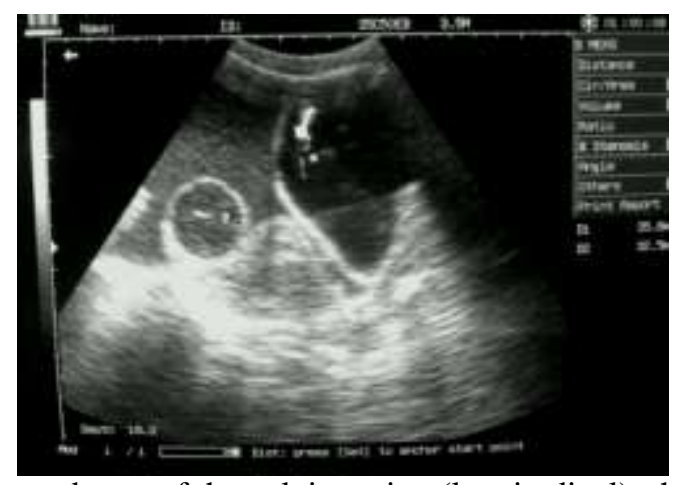

Figure1. Transabdominal ultrasound scan of the pelvic region (longitudinal), showing the oval-shaped hypoechoic mass surrounded bymassive ascites with internal echoes.

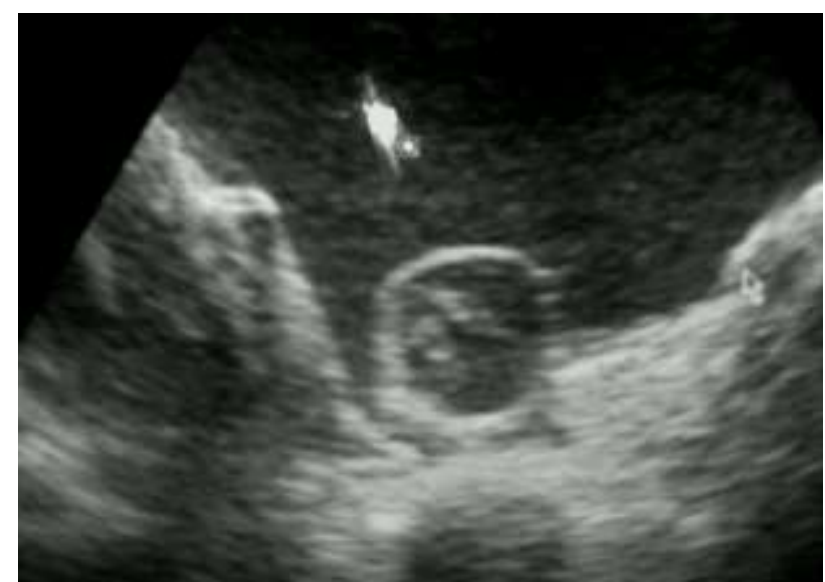

Figure 2 Zoomed image of the hypoechoic mass showing two nodules within. 


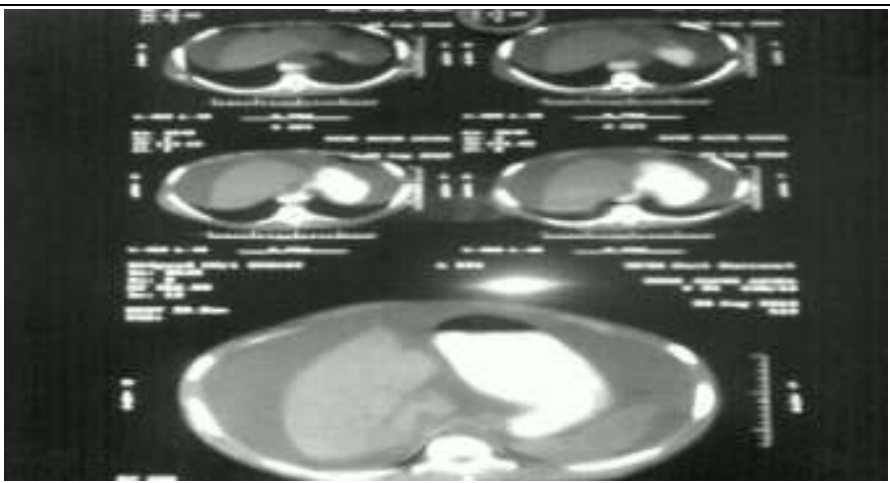

Figure 3 Axial Computed tomographic (CT) of the upper abdomen showing massive ascites.

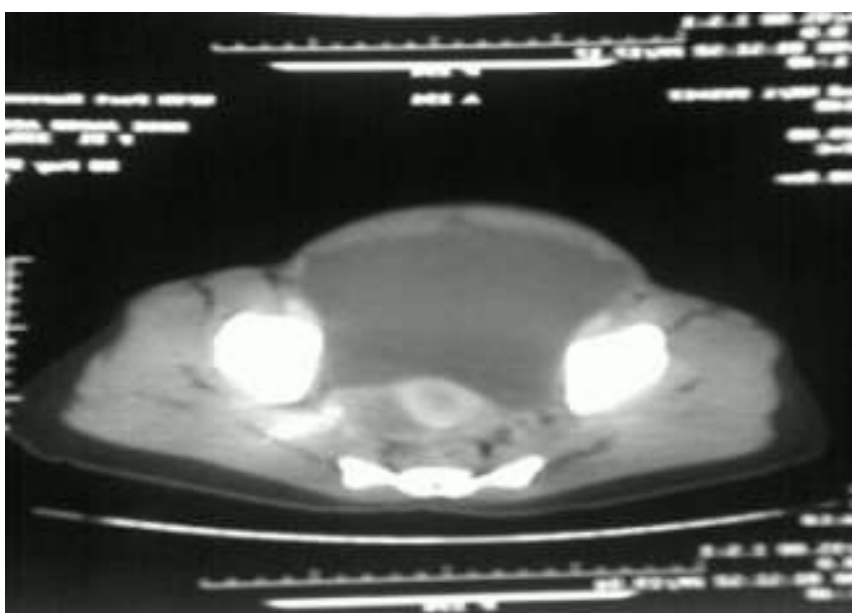

Figure 4 Axial Computed tomographic (CT) image of the of the pelvis showing the bladder and the uterus anterior to the rectum and massive ascites most anteriorly.

\section{Conclusion}

Endometriosis should be ruled out in all women in the child bearing age presenting with ascites. The case presented would have benefitted from MRI from the outset. It is the best modality in most of the sites of endometriosis and it is indicated when the US and CT findings are inconclusive. We hope this case presentation will help the referring gynaecologists make a right choice of imaging modality and the radiologist to apply the best techniques and to be abreast with the typical radiological findings to aid accurate diagnosis of endometriosis. Ultimately, patients suffering from this chronic recurring disease will receive prompt and accurate diagnosis.

\section{References}

[1] Anastasia Ussia, M D, George Betsas, M D, Roberta Corona, M D, Carlo De Cicco, M D, and Philippe R konincky, M D. Pathophysiology of cyclic Hemorrhagic Ascites and Endometriosis, The Journal of Minimally Invasive Gynecology 15(6), 2008, $677-681$.

[2] Ankur Lodha, Thomas Klein, Diana Elish, Regina Tarkovsky. Endometriosis : A Rare Presentation as Hemorrhagic Ascites,48 Practical Gastroenterology, 2008.

[3] Brews A. Endometriosis including endometriosis of the diaphragm in meigs syndrome, Proceeding of the Royal Society of Medicine $10,1954,309-312$.

[4] E .C Cheong, D T H Lim. Massive Ascites - An uncommon presentation of endometriosis, Singapore Medical Journal, 44(2), 2003, $098-100$.

[5] Yoshikawa H, Jimbo H, Okada S, Matsumoto K, onda T, Yasugi T, Taketani Y. Prevalence of Endometriosis in Ovarian Cancer, Gynecologic and Obstetric Investigation, 50(1), 2000, 11 - 17.

[6] Van Gorp T, Amant F, Neven P, Vergote I, Moerman P. Endometriosis and the developement of malignant tumour of the pelvis. A review of the literature, Best Practice \& Research Clinical Obstetrics \& Gynaecology, 18(2), 2004, 349-71.

[7] Ian S Fraser, A O, M D, Franzcog, Crei. Recognising, understanding and managing endometriosis, Journal of Human Reproductive Science, 1(2), 2008, $56-64$.

[8] Ivo Brosens, M D, FRCOG, Patrick Puttemans, M D, Rudi Campo, M D, Stephen Gordts, M D, Karen Kinkel, M D. Diagnosis of endometriosis: Pelvic endoscopy and imaging techniques, Best Practice and Research Clinical Obstetric and Gynaecology, 18(2), 2004, $285-303$

[9] Bazot M, Malzy P, Cortez A, Roseau G, Amouyal P, Dorai E. Accuracy of transvaginal sonography and rectal endoscopic sonography in the diagnosis of deep infiltrating endometriosis, Ultrasound Obstetric and Gynecology, 30(7), 2007,994 - 1001.

[10] Dumontier I, Chapron C, Chaussade S, Dubuisson J.B. Utility of rectal endoscopic ultrasound for digestive involvement of pelvic endometriosis. Technique and results, Gynecology and Obstetric Fertility, 30(12), 2002, 979 - 84. 
[11] Roseau G, Dumontier I, Palazzo L, Chapron C, Dousset B, Chaussade S, Dubuisson J B, Couturier D. Rectosigmoid endometriosis: endoscopic ultrasound features and clinical implications, Endoscopy, 32(7), 2000, 525 - 30.

[12] Karen Kinkel, Kathrin A Frei, Corinne Balleyguies, Charles Chapron. Diagnosis of endometriosis with imaging: a review, European Radiology, 16, 2006, 285 - 298.

[13] Kurjak A, Kupesic S, Anic T, Kosuta D. Three - dimentional ultrasound and power Doppler improve the diagnosis of ovarian lesions, Gynecologic Oncology, 76(1), 2000, $28-32$.

[14] Patel M D, Feldstein V A, Chan D C, Lipson S D, Filly R A. Endometriomas : diagnostic performance of US, Radiology, 210, $1999,739-745$

[15] Linda C. Giudice, M D, Ph.D. Endometriosis, The New England Journal of Medicine, 362: 2010, 2389 - 2398.

[16] Bazot M, Lafont C, Ronzier R, Rosseau G, Thomassin Naggara I, Darai E. Diagnostic accuracy of physical examination, transvaginal sonography, rectal endoscopic sonography and magnetic resonance imaging to diagnose deep infiltrating endometriosis, Fertility and Sterility, 92(6), 2009, $1825-33$.

[17] Hottat N, Larrousse C, Anaf V, Noel J C, Matos C, Absil J, Metens T. Endometriosis: Contribution of 3.0T Pelvic MR imaging in pre-operative assessment - - Initial results, Radiology, 253(1), 2009, 126 - 34.

[18] H K Ha, Y T Lim, H S Kim, T S Suh, H H Song, S J Kim. Diagnosis of pelvic endometriosis: Fat-suppressed $\mathrm{T}_{1}$ weighted VS Conventional MR Images, American Journal of Roentgenology, 163, 127-131.

[19] Jung S I, Kim Y J, Jeon H J, Jeong K A. Deep infiltrating endometriosis: CT imaging evaluation, Journal of Computer Assisted Tomography, 34(3), 2010, $338-42$.

[20] Vassilieff M, Suaud O, Collet-Savoye C, Da Costa C, Marouteau-pasquier N, Belhiba H, Tuech J J, Marpeau L, Roman H. Computed tomography-based virtual colonoscopy: an examination useful for the choice of the surgical management of colorectal endometriosis, Gynecology, Obstetrics and Fertility, 39(6), 2011,339-45.

[21] Eunhye Yoo, MD, Joo Hee Kim, MD, Myeong-jin Kim, MD, Jeong- sik YU, MD, Jae-Joon Chung, MD, Hyung-sik Yoo, MD and Ki Whang Kim, MD. Greater and lesser omenta: Normal Anatomy and Pathological Processes, RadioGraphics, 27, 2007,707 720. 